\title{
Probing $\mathrm{PrP}^{\mathrm{Sc}}$ Structure Using Chemical Cross-Linking and Mass Spectrometry: Evidence of the Proximity of Gly90 Amino Termini in the PrP 27-30 Aggregate $^{\dagger}$
}

\author{
Bruce Onisko, ${ }^{\ddagger}$ Esteban Guitián Fernández, ${ }^{\S}$ María Louro Freire, ${ }^{\prime}$ Anja Schwarz, ${ }^{\perp}$ Michael Baier, ${ }^{\perp}$ Félix Camiña, ${ }^{@}$ \\ Javier Rodríguez García, ${ }^{\circledR}$ Santiago Rodríguez-Segade Villamarín, ${ }^{\circledR}$ and Jesús R. Requena*,II \\ Western Regional Research Center, United States Department of Agriculture, Albany, California 94710, Mass Spectrometry \\ Unit, Prion Research Unit, Departments of Microbiology and Medicine, School of Medicine, and Department of Biochemistry, \\ School of Pharmacy, University of Santiago, Santiago, Spain, and Robert Koch Institute, Berlin, Germany
}

Received January 27, 2005; Revised Manuscript Received May 25, 2005

\begin{abstract}
Elucidation of the structure of $\mathrm{PrP}^{\mathrm{Sc}}$ continues to be one of the most important and difficult challenges in prion research. This task, essential for gaining an understanding of the basis of prion infectivity, has been hampered by the insoluble, aggregated nature of this molecule. We used a combination of chemical cross-linking, proteolytic digestion, and mass spectrometry (MALDI-TOF and nanoLC-ESI-QqTOF), in an attempt to gain structural information about PrP 27-30 purified from the brains of Syrian hamsters infected with scrapie. The rationale of this approach is to identify pairs of specific amino acid residues that are close enough to each other to react with a bifunctional reagent of a given chain length. We cross-linked PrP 27-30 with the amino-specific reagent bis(sulfosuccinimidyl) suberate $\left(\mathrm{BS}^{3}\right)$, obtaining dimers, trimers, and higher-order oligomers that were separated by SDS-PAGE. In-gel digestion followed by mass spectrometric analysis showed that $\mathrm{BS}^{3}$ reacted preferentially with Gly90. A cross-link involving two Gly90 amino termini was found in cross-linked PrP 27-30 dimers, but not in intramolecularly crosslinked monomers or control samples. This observation indicates the spatial proximity of Gly90 amino termini in PrP 27-30 fibrils. The Gly90-Gly90 cross-link is consistent with a recent model of PrP 2730 , based on electron crystallographic data, featuring a fiber composed of stacked trimers of PrP monomers; specifically, it is compatible with cross-linking of monomers stacked vertically along the fiber axis but not those adjacent to each other horizontally in the trimeric building block. Our results constitute the first measured distance constraint in $\mathrm{PrP}^{\mathrm{Sc}}$.
\end{abstract}

Prions were originally defined as "proteinaceous infectious particles", and very substantial and diverse evidence has accumulated to suggest that, indeed, their only component might be an intrinsically infectious protein (2). Transmissible spongiform encephalopathies (TSEs), ${ }^{1}$ a group of fatal neurodegenerative diseases affecting humans and animals, are associated with a prion, termed PrP (prion protein) (26). $\mathrm{PrP}$ can exhibit two conformations, $\mathrm{PrP}^{\mathrm{C}}$ and $\mathrm{PrP}^{\mathrm{Sc}}$. The latter is associated with TSEs, and PrP showing the physi-

$\doteqdot$ This work was supported by a research grant from the Spanish Ministry of Science and Technology (EET 2001-4861).

* To whom correspondence should be addressed: Prion Research Unit, Department of Microbiology, School of Medicine, Rúa de S. Francisco s/n, Santiago de Compostela 15782, Galiza, Spain. Phone: +34-981-563100, ext. 12255. Fax: +34-981-582642. E-mail: requenaj@usc.es.

$\checkmark$ United States Department of Agriculture.

$\S$ Mass Spectrometry Unit, University of Santiago.

" School of Medicine, University of Santiago.

${ }^{\perp}$ Robert Koch Institute.

@ School of Pharmacy, University of Santiago.

${ }^{1}$ Abbreviations: $\mathrm{BS}^{3}$, bis(sulfosuccinimidyl) suberate; $\alpha$-CHC, $\alpha$-cyano-4-hydroxycinnamic acid; GPI, glycosylinositol phospholipid; lys C, lysyl endopeptidase; PK, proteinase K; PNGase F, $N$-glycosidase F; PrP, prion protein; rms, root-mean-square; ShaPrP, Syrian hamster prion protein; TSEs, transmissible spongiform encephalopathies. Abbreviations for peptide sequence ions ( $\mathrm{a}, \mathrm{b}, \mathrm{c}, \mathrm{x}, \mathrm{y}$, and $\mathrm{z}$ ) follow the nomenclature of Roepstorff and Fohlman (1). cochemical characteristics of $\mathrm{PrP}^{\mathrm{Sc}}$ is isolated as the main and most probably only component of the TSE infectious agent. $\operatorname{PrP}^{\mathrm{C}}$ can convert into $\operatorname{PrP}^{\mathrm{Sc}}$ in the presence of preformed $\mathrm{PrP}^{\mathrm{Sc}}$, through a poorly understood molecular process $(6,7)$. The structure of $\operatorname{PrP}^{\mathrm{C}}$ has been characterized by NMR (8), but that of $\mathrm{PrP}^{\mathrm{Sc}}$ is largely unknown, as its insolubility in nondenaturing solvents has seriously hampered analytical efforts. It is known, however, that $\operatorname{PrP}^{\mathrm{Sc}}$ and $\mathrm{PrP}^{\mathrm{C}}$ differ with respect to secondary, tertiary, and quaternary structure $(3,9)$. No covalent differences have been detected between the two molecules (10), although the possibility that post-translational modifications of a small set of $\operatorname{PrP}^{\mathrm{C}}$ molecules could trigger structural changes relevant to initiation of conversion to $\mathrm{PrP}^{\mathrm{Sc}}$ cannot be ruled out (11). Studies using Fourier transform infrared spectroscopy indicate that $\mathrm{PrP}^{\mathrm{Sc}}$ contains an increased fraction of $\beta$-sheet and decreased fractions of $\alpha$-helix and random coil with respect to $\operatorname{PrP}^{\mathrm{C}}$ $(3,9) \cdot \mathrm{PrP}^{\mathrm{C}}$ is a monomeric protein anchored to the cell membrane through a glycosylinositol phospholipid (GPI) moiety. $\mathrm{PrP}^{\mathrm{Sc}}$ is isolated as an aggregate, and is partially resistant to proteinase $\mathrm{K}(\mathrm{PK})$, that trims an amino-terminal segment of the protein, generating a well-defined resistant core termed $\operatorname{PrP} 27-30$, whereas $\operatorname{PrP}^{\mathrm{C}}$ is rapidly degraded by PK (3). PrP 27-30 retains the infectious character, and hence the essential structural characteristics, of $\mathrm{PrP}^{\mathrm{Sc}}$, with 
the trimmed amino-terminal domain probably consisting of a highly flexible tail as seen in $\operatorname{PrP}^{\mathrm{C}}$. Recently, a PK-sensitive subfraction of $\mathrm{PrP}^{\mathrm{Sc}}$ has been described. This material shares structural features with PK-resistant $\mathrm{PrP}^{\mathrm{Pc}}$; namely, it shows enhanced reactivity toward certain monoclonal antibodies after treatment with guanidine (12). Whether PK-sensitive $\mathrm{PrP}^{\mathrm{Sc}}$ is just a less aggregated form of $\mathrm{PrP}^{\mathrm{Sc}}(13)$ and whether it is infectious remain to be seen. In the presence of detergent, PrP 27-30 further polymerizes to rod-shaped filaments with the tinctorial properties of amyloid (14).

Since the only difference between $\operatorname{PrP}^{\mathrm{Pc}}$ and $\operatorname{PrP}^{\mathrm{C}}$ appears to be conformational, it has been reasoned that the unique transmissible character of $\mathrm{PrP}^{\mathrm{Sc}}$ must be a consequence of its particular conformation. Therefore, a knowledge of the structure of $\mathrm{PrP}^{\mathrm{Sc}}$ is essential in understanding its transmissibility, the core of the prion concept.

Recent reports have demonstrated the utility of an approach based on combining protein cross-linking, proteolytic digestion, and mass spectrometry for obtaining information regarding the three-dimensional structures of proteins (1517). The approach involves cross-linking of specific reactive groups using a bifunctional reagent, proteolytic digestion, and analysis of the digest by mass spectrometry in identifying cross-linked peptides and, hence, linkage sites. With polymeric proteins, monomers, dimers, trimers, etc., formed as a consequence of intra- or intermolecular cross-linking reactions can be separated prior to digestion. The length of the cross-linking reagent that is used determines distance constraints that provide information about the relative proximity of specific pairs of residues and, therefore, of the domains or protein subunits in which they are located. Using model proteins of known structure, it has been claimed that the method can be used, in conjunction with threading programs, to identify the fold of a protein (15). The method has been used to obtain valuable information about key features of unknown tertiary and quaternary structures of proteins (15-24). One particularly relevant example of the use of a variation of this approach was reported by Egnaczyk et al. (24), who used it to probe the quaternary structure of $\beta$-amyloid fibrils. We reasoned that such an approach might be useful in obtaining information about the structure of $\mathrm{PrP}^{\mathrm{Sc}}$. We report here the successful application of this methodology to PrP 27-30 and discuss the implications of the observed distance constraints.

\section{MATERIALS AND METHODS}

Reagents. The water-soluble, amino group-selective bifunctional cross-linking reagent bis(sulfosuccinimidyl) suberate $\left(\mathrm{BS}^{3}\right)$ was purchased from Pierce (Rockford, IL). $\alpha$-Cyano-4-hydroxycinnamic acid $(\alpha-C H C)$ was from Bruker Daltonics (Billerica, MA). Achromobacter lysyl endopeptidase (lys C) was obtained from Wako (Osaka, Japan). An $N$-Glycosidase F (PNGase F) deglycosylation kit was obtained from New England Biolabs (Beverly, MA). Recombinant Syrian hamster PrP(90-231) was obtained from InPro Biotechnology (South San Francisco, CA). All other reagents were from Sigma-Aldrich.

Syrian Hamster PrP 27-30. PrP 27-30 was isolated as described previously (25) from brains of terminally ill Syrian hamsters infected intracranially with the $263 \mathrm{~K}$ strain of scrapie. Its purity and approximate concentration were assessed by SDS-PAGE and Coomassie blue staining. PrP $27-30$ was suspended immediately before being used in $1 \%$ sarkosyl at an approximate concentration of $0.2 \mu \mathrm{g} / \mu \mathrm{L}$ by sonication with a 4710 Series probe ultrasonics homogenizer (Cole Parmer, Chicago, IL).

Cross-Linking Reactions. PrP 27-30, $\sim 2-10 \mu \mathrm{g}$, was cross-linked in $100 \mathrm{mM}$ phosphate buffer $(\mathrm{pH}$ 7.2) at a concentration of $0.067 \mu \mathrm{g} / \mu \mathrm{L} ; \mathrm{BS}^{3}$ was added from a freshly prepared $10 \mathrm{mM}$ stock solution in $5 \mathrm{mM}$ sodium acetate $(\mathrm{pH}$ 5) to a final concentration of $1.7 \mathrm{mM}$, and allowed to react with the protein for $30 \mathrm{~min}$ at room temperature. The reaction was then terminated by addition of $1 \mathrm{M}$ lysine $(\mathrm{pH}$ 7.2) to a final concentration of $140 \mathrm{mM}$ and further incubation at room temperature for $15 \mathrm{~min}$. Control samples were treated in the same way, except that a $5 \mathrm{mM}$ sodium acetate solution $(\mathrm{pH} 5)$ was added instead of a $\mathrm{BS}^{3}$ solution.

Deglycosylation and Electrophoretic Separation. Crosslinked or control protein was precipitated by centrifugation at $14000 \mathrm{rpm}$ in a tabletop centrifuge for $45 \mathrm{~min}$; supernatants were carefully aspirated and discarded, and pellets were rinsed with $200 \mu \mathrm{L}$ of $85 \%$ methanol. Pellets were then denatured and deglycosylated with $3 \mu \mathrm{L}$ of a PNGase solution at $37^{\circ} \mathrm{C}$ for $1 \mathrm{~h}$, according to the manufacturer's instructions. Reaction mixtures were then diluted with an equal volume of reducing Laemmli sample buffer, boiled for $10 \mathrm{~min}$, and subjected to SDS-PAGE (26) using $12 \%$ gels. Protein bands were stained with Coomassie blue.

In-Gel Proteolytic Digestion. For samples analyzed by MALDI-TOF, protein bands were carefully excised with a razor blade, and then reduced, alkylated, and digested ingel at an approximate mass ratio of 1:10 with trypsin (sequencing grade, modified, Promega, Madison, WI) or lys $\mathrm{C}$ to PrP, according to the procedure of Shevchenko et al. (27) with slight modifications. Briefly, bands were cut into $1 \mathrm{~mm}^{3}$ pieces, placed in an Eppendorf tube, washed with water, and dehydrated with $200 \mu \mathrm{L}$ of acetonitrile for 15 min using mild agitation. Acetonitrile was removed, and the gel pieces were dried in vacuo (SpeedVac, Savant, Farmingdale, CA); a volume of $30 \mu \mathrm{L}$ of $10 \mathrm{mM}$ DTT in $25 \mathrm{mM}$ $\mathrm{NH}_{4} \mathrm{HCO}_{3}$ was added, and reduction was carried out at 56 ${ }^{\circ} \mathrm{C}$ for $30 \mathrm{~min}$. The solvent was then removed and, after dehydration of gel pieces with acetonitrile as described, replaced with $30 \mu \mathrm{L}$ of $55 \mathrm{mM}$ iodoacetamide. Alkylation was carried on in the dark at room temperature for $20 \mathrm{~min}$. The solvent was then removed, and gel pieces were washed with $25 \mathrm{mM} \mathrm{NH}_{4} \mathrm{HCO}_{3}$, dehydrated with acetonitrile, and rehydrated on ice by addition of $20 \mu \mathrm{L}$ of $25 \mathrm{mM} \mathrm{NH}_{4} \mathrm{HCO}_{3}$ containing $15 \mathrm{ng} / \mu \mathrm{L}$ trypsin or lys C. After $40 \mathrm{~min}, 30 \mu \mathrm{L}$ of $25 \mathrm{mM} \mathrm{NH} \mathrm{HCO}_{3}$ was added to cover the gel pieces and samples were incubated overnight at $37^{\circ} \mathrm{C}$. Digested samples were briefly centrifuged, and the supernatant was collected. Gel pieces were then extracted with $20 \mu \mathrm{L}$ of $25 \mathrm{mM} \mathrm{NH}_{4}$ $\mathrm{HCO}_{3}$ with sonication for $10 \mathrm{~min}$. The solvent was then recovered and replaced with $20 \mu \mathrm{L}$ of $0.1 \%$ trifluoroacetic acid (TFA). The extracts and the digestion solution were pooled and dried in vacuo. Peptides were redissolved in 10 $\mu \mathrm{L}$ of $0.1 \%$ TFA and $50 \%$ acetonitrile.

For samples analyzed by nanoLC-MS/MS, protein spots were excised from gels and then processed in a DigestPro (INTAVIS Bioanalytical Instruments AG, Bergish Gladbach, Germany). Following washing, reduction with DTT, alkylation with iodoacetamide, and in-gel digestion (porcine 
trypsin, Princeton Separations, Adelphia, NJ), the peptides were eluted into a 96-well collection plate with $60 \mu \mathrm{L}$ of $10 \%$ formic acid containing $0.1 \%$ trifluoroacetic acid.

$M A L D I$. A $2 \mu \mathrm{L}$ portion of protein digest was mixed with an equal volume of a saturated solution of $\alpha-\mathrm{CHC}$ in acetonitrile and $0.1 \%$ aqueous TFA (1:2). One microliter of the mixture was spotted onto a Bruker sample plate, allowed to air-dry, and analyzed using a Bruker Autoflex MALDI instrument in reflectron mode. The laser frequency was 25 $\mathrm{Hz}$; pulsed ion extraction was set at a value of $140-150 \mathrm{~ns}$. Repeated laser shots, typically $25-30$, were averaged.

Nanospray LC-MS/MS. NanoLC-ESI-MS/MS was carried out with an Applied Biosystems (ABI/MDS Sciex, Toronto, ON) Model QStar Pulsar apparatus equipped with a Proxeon Biosystems (Odense, Denmark) nano-electrospray source. In-gel digest $(20 \mu \mathrm{L})$ was loaded automatically onto a C-18 trap cartridge and chromatographed on a reversedphase column (Vydac 238EV5.07515, $75 \mu \mathrm{m} \times 150 \mathrm{~mm}$; Hesperia, CA) fitted at the effluent end with a coated spray tip (FS360-50-5-CE, New Objective Inc., Woburn, MA). An LC Packings nano-flow LC system (Dionex, Sunnyvale, CA) with an autosampler, a column switching device, a loading pump, and a nano-flow solvent delivery system was used to elute the column. Elution solvents were as follows: A, 0.5\% acetic acid in water; and B, $80 \%$ acetonitrile and $0.5 \%$ acetic acid. Samples were eluted at a rate of $250 \mathrm{~nL} / \mathrm{min}$ with the following gradient profile: $2 \% \mathrm{~B}$ at $0 \mathrm{~min}$ to $80 \% \mathrm{~B}$ in a 15 min linear gradient, held at $80 \%$ B for $5 \mathrm{~min}$, and then back to $2 \% \mathrm{~B}$ for $10 \mathrm{~min}$. The QStar Pulsar apparatus was externally calibrated daily and operated at a resolution of $>10000$. The acquisition cycle time of $6 \mathrm{~s}$ consisted of a single $1 \mathrm{~s}$ MS "survey" scan followed by a $5 \mathrm{~s}$ MS/MS scan. Ions between $\mathrm{m} / \mathrm{z}, 400$ and 1000 of charge states between +2 to +5 having intensities greater than 40 counts in the survey scan were selected for fragmentation. The dynamic exclusion window was set to always exclude previously fragmented masses. The collision energy optimized for charge state and $\mathrm{m} / \mathrm{z}$ was automatically selected by the Analyst QS software after adjusting parameters to obtain satisfactory fragmentation of Glu fibrinogen peptide $(+2)$ and $\mathrm{ACTH}(+3$ and +4$)$. Nitrogen was used as the collision gas, and the pressure in the collision cell ranged from $3 \times$ $10^{-6}$ to $6 \times 10^{-6}$ Torr.

Data Analysis. LC-MS/MS data were internally calibrated using trypsin autolysis peptides whose identities were confirmed by MS/MS. The MS/MS data were submitted to Mascot (http://www.matrixscience.com/search_form_select.html) to assist in the assignment of unmodified peptides present in the digest (for these peptides, an rms mass error of $10-15 \mathrm{ppm}$ was observed). Next, the calibrated TOFMS survey scans were processed with the "LCMS Reconstruct" tool in Analyst. The output is a list of peptide molecular weights calculated by deconvolution of multiple charge states and then identification of the monoisotopic ${ }^{12} \mathrm{C}$ species. Lists of peaks present in spectra of monomeric and dimeric cross-linked samples were compiled. Using custom software written in-house, those peaks also present in spectra of control digests of PrP 27-30 were deleted from crosslinked sample peak lists using a $20 \mathrm{ppm}$ mass tolerance. The resulting experimental list of monoisotopic molecular weights was compared to a list of theoretical cross-linked and modified peptides predicted from the sequence of ShaPrP-

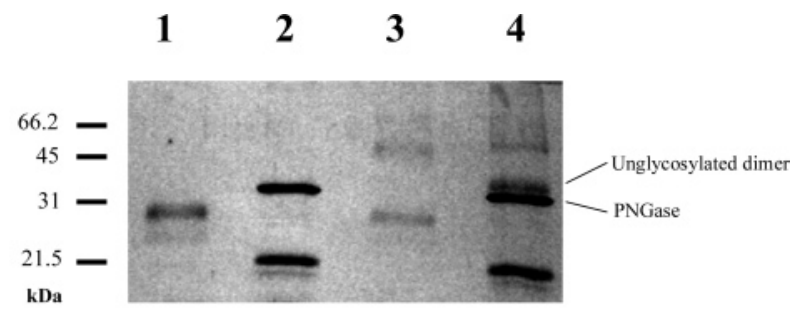

$\begin{array}{lllll}\text { BS }^{3} & - & - & + & + \\ \text { PNGase } & - & + & - & +\end{array}$

Figure 1: Cross-linking of $\operatorname{PrP} 27-30$ by $\mathrm{BS}^{3}$. PrP 27-30 was treated with $1.7 \mathrm{mM} \mathrm{BS}{ }^{3}$ at $37^{\circ} \mathrm{C}$ for $30 \mathrm{~min}$ (see Materials and Methods). The reaction was quenched with lysine and PrP 27-30 submitted to SDS-PAGE with or without previous deglycosylation with PNGase F. The gel was stained with Coomassie blue: lane 1, control PrP 27-30; lane 2, deglycosylated control PrP 27-30; lane $3, \mathrm{BS}^{3}$-treated PrP 27-30; and lane 4, deglycosylated $\mathrm{BS}^{3}$-treated PrP 27-30.

(90-231) using the X-link search tool of GPMaw (http:// welcome.to/gpmaw).

For MALDI analysis, peaks were externally calibrated using a peptide mix standard (angiotensin II, angiotensin I, Substance P, Bombesin, ACTH clip 1-17, ACTH clip 1839, and Somatostatin 28 from Bruker); identified major tryptic fragments predicted from the sequence of ShaPrP(90-231) were then used to internally calibrate unknown peaks.

\section{RESULTS}

Treatment of PrP 27-30 with $\mathrm{BS}^{3}$ resulted in crosslinking, documented by the appearance of new bands on polyacrylamide gels corresponding to dimers, trimers, and higher-order multimers of PrP 27-30 (Figure 1). Dimers and trimers were also generated at lower concentrations of $\mathrm{BS}^{3}$ $(0.17 \mathrm{mM})$, although oligomer bands were less abundant (data not shown). Treatment of isolated PrP 27-30 with PNGase removes Asn-linked oligosaccharides and results in a major band with a molecular mass of $21000 \mathrm{Da}$ (Figure 1). An 8000-9000 Da loss is consistent with removal of both Asn-linked oligosaccharides and suggests that the isolated PrP 27-30 has oligosaccharides present at both known sites, Asn181 and Asn197. Treatment of cross-linked PrP 27-30 with PNGase resulted in analogous mass losses for the monomer, dimer, and trimer bands (Figure 1).

A control experiment in which the order of addition of the cross-linking and quenching reagents was reversed resulted in no cross-linking (data not shown). This control rules out the possibility that the cross-linked species are artifacts arising from further $\mathrm{BS}^{3}$ reaction during sample manipulation after the native $\operatorname{PrP}^{\mathrm{Sc}}$ conformation has been lost. Separate control reactions used recombinant Syrian hamster PrP (residues 90-231) at twice the protein concentration $(0.14 \mu \mathrm{g} / \mu \mathrm{L})$ as with isolated $\operatorname{PrP} 27-30$ and $\mathrm{BS}^{3}$, at either 0.22 or $0.043 \mathrm{mM}$ for $1 \mathrm{~h}$ and at either room temperature or $0{ }^{\circ} \mathrm{C}$. No dimer band was seen by SDSPAGE in any of the control reactions, although nanoLCQqTOF analysis revealed considerable $\mathrm{BS}^{3}$ addition in all cases (data not shown). These control experiments strongly suggest that the dimers formed with PrP 27-30 are not the result of random collisions in solution between PrP molecules. 


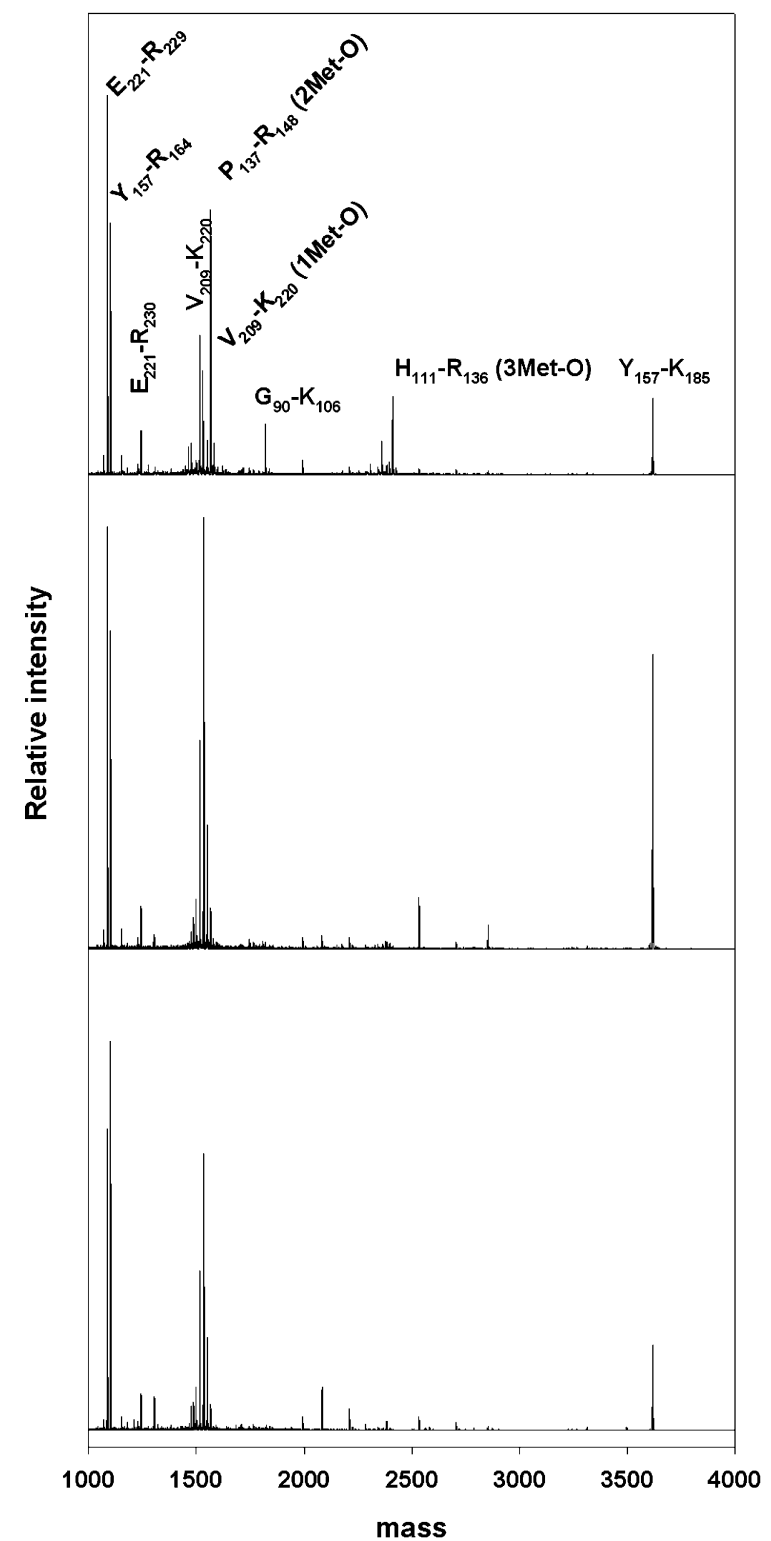

FIGURE 2: Mass spectra of the cross-linked monomer and dimer and of control deglycosylated PrP 27-30. Bands were excised from gels and digested in gel with trypsin overnight at $37^{\circ} \mathrm{C}$. Peptides were extracted, dried in vacuo, and analyzed by MALDI-TOF as described. Spectra of control, monomer, and dimer digests (top, middle, and bottom panels, respectively). The main identified peaks are indicated. For a more comprehensive list of peaks, see Table $\mathrm{S} 1$ of the Supporting Information.

Tryptic digests of PrP 27-30, cross-linked monomer, and dimer bands from SDS-PAGE were analyzed by MALDITOF (Figure 2 and Figure S1 of the Supporting Information) and nanoLC-ESI-QTOF mass spectrometry (Figure S2 of the Supporting Information). Spectra from the cross-linked monomer and dimer bands are very similar to those of control digests of PrP 27-30. Some variability was observed in the relative intensities of some peaks due to variable oxidation of peptides containing methionine residues. Also, some variability in the extent of cleavage by trypsin at KP sites was observed. Good sequence coverage $(88 \%)$ of the expected tryptic peptides was observed in all samples and by both techniques (Table S1 of the Supporting Information summarizing ESI data, and Figure S3 of the Supporting Information showing partial ShaPrP sequence); the rms mass

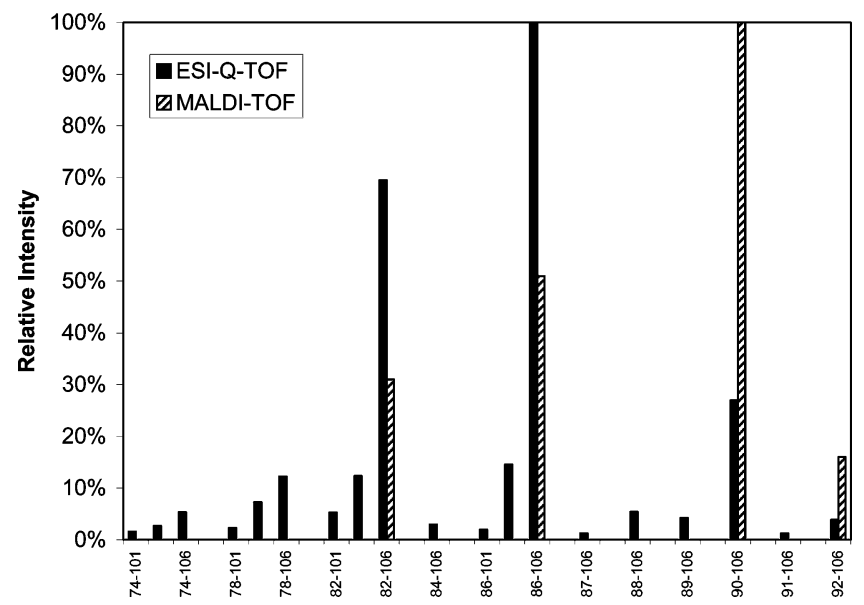

FIGURE 3: Comparison of the relative intensities of amino-terminal tryptic peptides from PrP 27-30 identified by MALDI-TOF and nanoLC-ESI-QqTOF mass spectrometry.

error for the peptides listed was $12 \mathrm{ppm}$, and the identity of the indicated sequences was confirmed by MS/MS (data not shown).

Proteolytic activity of proteinase $\mathrm{K}$ used during purification of PrP 27-30 generates a mixture of amino-terminally truncated molecular species. Amino-terminal peptides formed by tryptic digestion of these species were identified by MALDI-TOF and nanoLC-ESI-QTOF mass spectrometry. Relative abundances of these amino-terminal tryptic peptides are shown in Figure 3. Interpretation is somewhat complicated because of limited tryptic cleavage at Lys101/Pro102 and at Lys104/Pro105. Analysis of the ESI data shows three abundant amino termini (Gly82, Gly86, and Gly90), and several other amino termini of low abundance. The rms mass error for the 19 peptides found by ESI was $12 \mathrm{ppm}$, and the identity of the indicated sequences was confirmed by MS/ MS (data not shown). The MALDI results are quite similar. Four abundant amino termini were seen (Gly82, Gly86, Gly90, and Gly92). ESI data show the amino terminus at Gly 86 to be most abundant, whereas Gly90 was found to be most abundant by MALDI. This apparent discrepancy is readily explained by the intrinsic differences in ionization modes characteristic of ESI and MALDI, and by the fact that the C-18 trap cartridge used for sample preparation prior to ESI may result in losses of specific peptides, particularly small, less hydrophobic ones. In fact, pretreatment of samples with C-18 ZipTips (Millipore, Bedford, MA) prior to MALDI substantially decreased the yield of all amino-terminal peptides, but more particularly of those starting at Gly82 and Gly86 (data not shown).

Comparison of the relative abundances of the aminoterminal peptides found in the cross-linked monomer and dimer bands to the amounts seen in PrP 27-30 reveals a marked, consistent decrease in the abundance of all the abundant amino-terminal species in the cross-linked samples (Figure 2 and Figure S1 and Table S1 of the Supporting Information). This effect was common to spectra of both trypsin and lys $\mathrm{C}$ digests (data not shown). While neither ionization method can yield quantitative data without the use of isotopically labeled standards, these results strongly suggest that amino-terminal amino groups extensively react with $\mathrm{BS}^{3}$. The decreased abundances of the amino-terminal species in the cross-linked samples are also indicative of 
Table 1: Theoretical and Experimental Molecular Weights of Modified Tryptic Peptides from PrP 27-30 Control and PrP 27-30 Cross-Linked with $\mathrm{BS}^{3}$ (monomer and dimer bands)

\begin{tabular}{|c|c|c|c|c|c|c|c|}
\hline \multirow[b]{2}{*}{ species } & \multirow[b]{2}{*}{ theory MW } & \multicolumn{3}{|c|}{$\operatorname{PrP} 27-30 \mathrm{BS}^{3}$ dimer } & \multicolumn{3}{|c|}{ PrP $27-30 \mathrm{BS}^{3}$ monomer } \\
\hline & & observed MW & error $(\mathrm{ppm})$ & intensity (\%) & observed MW & error $(\mathrm{ppm})$ & intensity $(\%)$ \\
\hline $82-106+\mathrm{C}_{8} \mathrm{H}_{12} \mathrm{O}_{3}$ & 2752.32 & 2752.31 & 5 & 8 & 2752.37 & 16 & 7 \\
\hline $86-106+\mathrm{C}_{8} \mathrm{H}_{12} \mathrm{O}_{3}$ & 2333.13 & - & - & - & 2333.15 & 11 & 18 \\
\hline $90-106+\mathrm{C}_{8} \mathrm{H}_{12} \mathrm{O}_{3}{ }^{a}$ & 1975.99 & 1975.97 & 9 & 21 & 1975.97 & 9 & 37 \\
\hline $92-110+\mathrm{C}_{8} \mathrm{H}_{12} \mathrm{O}_{3}$ & 2265.13 & 2265.17 & 14 & 24 & - & - & - \\
\hline $221-229+\mathrm{C}_{8} \mathrm{H}_{12} \mathrm{O}_{3}{ }^{a}$ & 1243.53 & 1243.54 & 4 & 100 & 1243.54 & 4 & 100 \\
\hline $90-106+90-106+\mathrm{C}_{8} \mathrm{H}_{10} \mathrm{O}_{2}$ & 3777.88 & 3777.82 & 15 & 44 & - & - & - \\
\hline $90-106+86-101+\mathrm{C}_{8} \mathrm{H}_{10} \mathrm{O}_{2}$ & 3597.70 & 3597.72 & 6 & 9 & - & - & - \\
\hline $90-106+90-104+\mathrm{C}_{8} \mathrm{H}_{10} \mathrm{O}_{2}$ & 3552.74 & 3552.72 & 4 & 60 & - & - & - \\
\hline $90-106+90-101+\mathrm{C}_{8} \mathrm{H}_{10} \mathrm{O}_{2}{ }^{a}$ & 3240.56 & 3240.56 & 2 & 71 & - & - & - \\
\hline $91-106+92-101+\mathrm{C}_{8} \mathrm{H}_{10} \mathrm{O}_{2}$ & 2998.45 & 2998.43 & 8 & 42 & - & - & - \\
\hline
\end{tabular}

${ }^{a}$ Peptide structure confirmed by MS/MS.

exposed locations of the various amino termini allowing facile reaction with the ionic (charge of -2 at $\mathrm{pH} 7$ ) and somewhat large cross-linking reagent $(\mathrm{MW}=572.4)$. An extended conformation of $\mathrm{BS}^{3}$ would be $\sim 20 \AA$ long, and is shaped like a femur with $7.4 \AA$ diameter spheres at each end.

Direct evidence of the reaction of the amino termini with $\mathrm{BS}^{3}$ was also observed (Table 1). Four tryptic peptides were found as their suberic acid amides $\left(\mathrm{M}+\mathrm{C}_{8} \mathrm{H}_{12} \mathrm{O}_{3}\right)$, formed by reaction of $\mathrm{BS}^{3}$ with the amino terminus of the peptide followed by hydrolysis of the remaining $N$-hydroxysuccinimide ester that thus fails to react with a second amino group. The modified peptides were assigned to residues $82-106$, $86-106,90-106$, and $92-110$ which correspond to the four most abundant amino-terminally truncated PrP $27-30$ species (see Figure 3). The rms mass error for the six measurements (three from the dimer band and three from the monomer band from SDS-PAGE of cross-linked PrP 27-30) was $11 \mathrm{ppm}$. The identity of the indicated sequences was confirmed by MS/MS (data not shown). The collisionactivated dissociation (CAD) spectra of modified peptide 90-106 clearly show that suberic acid is linked via the amino terminus of the peptide and not via the $\epsilon$-amino group of either Lys101, Lys104, or Lys106 (data not shown). These results demonstrate that Gly82, Gly86, Gly90, and Gly92 are accessible to the cross-linking reagent and thus must be on the surface of $\operatorname{PrP} 27-30$ fibrils.

Tryptic peptide 221-229 was found as its suberic acid ester $\left(\mathrm{M}+\mathrm{C}_{8} \mathrm{H}_{12} \mathrm{O}_{3}\right)$, which is formed by reaction of $\mathrm{BS}^{3}$ with the phenolic group of a Tyr (28). The sequence of residues 221-229 contains two Tyr residues, and MS/MS of the +3 precursor of $m / z, 415.52$ clearly shows that Tyr226 (and not Tyr225) is present as its suberic acid ester. Fragment ions corresponding to y4, y5, y6, y7, and y8 were observed at $\mathrm{m} / \mathrm{z}$ (relative abundance) $666.30(18 \%), 829.36(38 \%)$, $900.40(14 \%), 1028.45(8 \%)$, and $1115.49(3 \%)$, respectively. The calculated $\mathrm{m} / \mathrm{z}$ of the $\mathrm{y} 4$ ion (510.23) for the isomeric derivative of Tyr225 was not observed. The same peptide derivative was found in both the monomer and dimer bands formed from reaction of $\operatorname{PrP} 27-30$ with $\mathrm{BS}^{3}$. It should be noted that the CAD spectra of ESQAYYDGRR and ESQAYZDGR (where $\mathrm{Z}$ is a tyrosine esterified with suberic acid) are extremely similar. This is because the additional mass corresponding to the presence of one arginine residue $\mathrm{C}_{6} \mathrm{H}_{12} \mathrm{~N}_{4} \mathrm{O}$ (156.101) is almost the same as the mass increase due to esterification of tyrosine with suberic acid $\mathrm{C}_{8} \mathrm{H}_{12} \mathrm{O}_{3}$
(156.079). Fortunately, y4 ions were observed which can readily distinguish these molecules (503.27 for ESQAYYDGRR and 666.31 for ESQAYZDGR). Furthermore, accurate mass data alone could be used to assign the spectra. For ESQAYYDGRR, the rms error for the observed y ion series was $7 \mathrm{ppm}$ for the correct sequence (and $32 \mathrm{ppm}$ for the alternate possibility). For ESQAYZDGR, the rms error for the observed y ion series was $6 \mathrm{ppm}$ for the correct sequence (and $19 \mathrm{ppm}$ for the alternate possibility).

Five intermolecular cross-linked species were also found (Table 1 and Figure 4) in the dimer bands from SDS-PAGE. None were found in the control or monomer bands, as expected. Three of the intermolecular cross-links arise by cross-linking two PrP 27-30 monomers which both have Gly90 amino termini; the three species observed differ in the location of their carboxy termini due to limited tryptic cleavage at Lys101/Pro102 and at Lys104/Pro105. The other intermolecular cross-linked species arise from cross-linking PrP 27-30 monomers with amino termini at Gly90 and Gly86, or from cross-linking PrP 27-30 monomers with amino termini at Gly92 and Gly91, respectively. The rms mass error for the five cross-linked species found by ESI was 7 ppm. Furthermore, the theoretical masses of individual isotopomers, and the distribution thereof, match theoretical data quite well (see Figure 5 for TOF-MS data for the +5 charge state ion of 90-106 + 90-101 $+\mathrm{C}_{8} \mathrm{H}_{10} \mathrm{O}_{2}$ ). The most abundant cross-link corresponding to 90-106 + $\mathrm{C}_{8} \mathrm{H}_{10} \mathrm{O}_{2}+90-101$ was confirmed by MS/MS (Figure S4 of the Supporting Information). The observed fragmentation is fully consistent with the proposed structure. Good sequence coverage was obtained for the proline-containing peptide. Internal ions corresponding to PSK and PSKP were also seen, as were the expected immonium ions for tryptophan, histidine, and glutamine. Due to the difficulty in obtaining good quality MS/MS data on small amounts of relatively large molecules (MW $=3000-4000$ ), only the most abundant cross-link was confirmed by MS/MS.

Analysis of the dimer sample by MALDI confirmed the existence of the intermolecular species that is formed by cross-linking two PrP 27-30 monomers which both have Gly90 amino termini. The evidence was a peak with a monoisotopic mass of 2703.18 (theoretical mass of 2703.23, error $=18 \mathrm{ppm}$ ), corresponding to an intermolecular crosslink involving two 90-101 amino-terminal peptides, seen in the lys $\mathrm{C}$ digest of cross-linked dimeric samples, but not in cross-linked monomers or controls (Figure 6). 

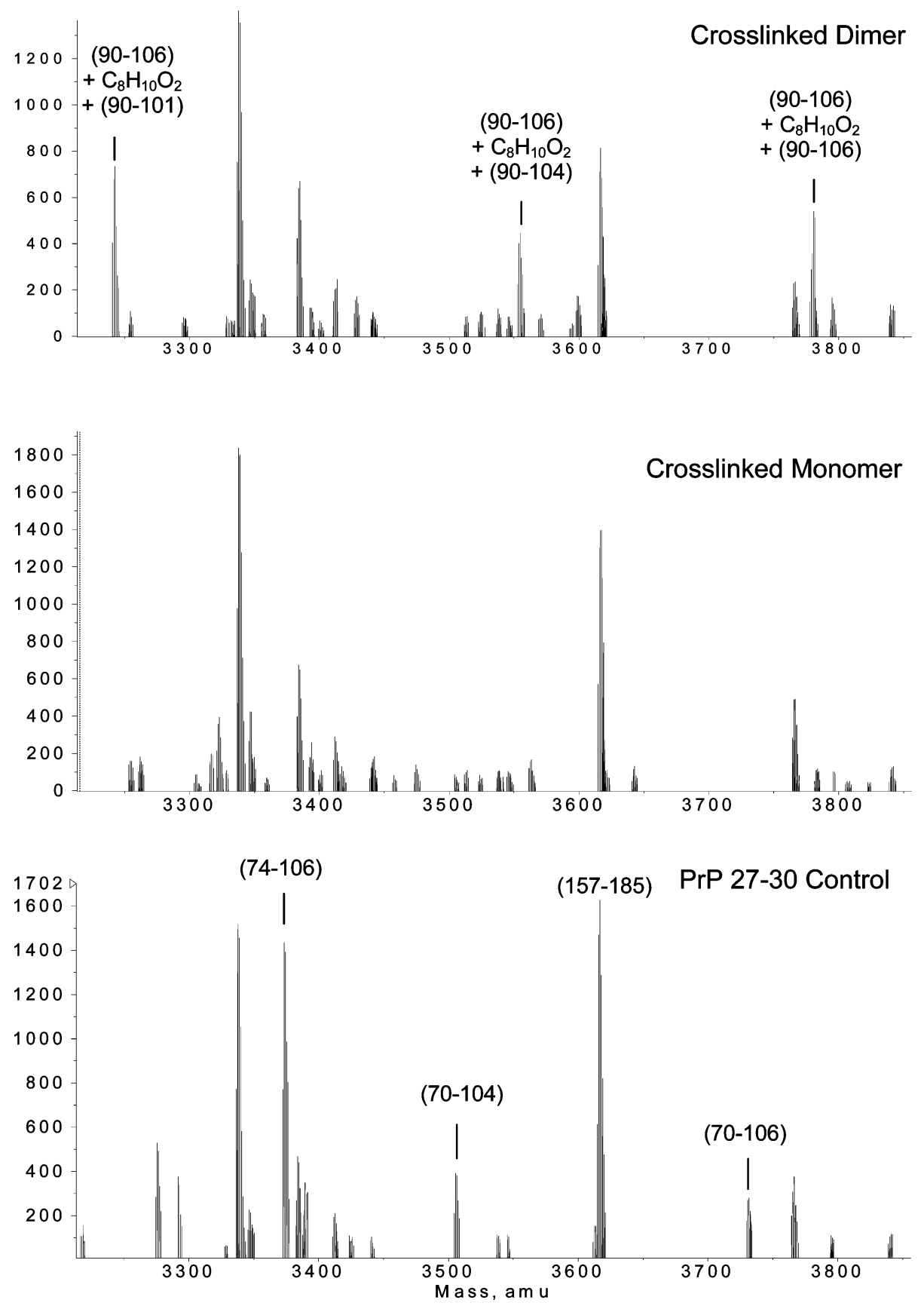

FIGURE 4: Analysis by nanoLC-ESI-QqTOF mass spectrometry of cross-linked peptides from trypsin digests of the PrP 27-30 control

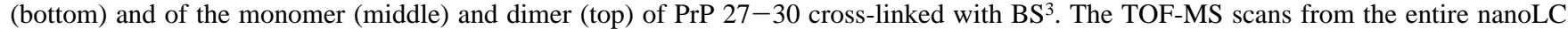
chromatogram were summed, and then the series of multiply charged peaks were transformed from the $\mathrm{m} / \mathrm{z}$ domain to the mass domain which is plotted on the $x$ axis.

The observation of unmodified amino-terminal peptides (Table S1 of the Supporting Information) and their suberic acid amides (Table 1) in the tryptic digests of cross-linked PrP 27-30 dimers infers that some of the dimers are crosslinked at sites other than via their amino termini. Comparing the relative abundance of the 90-106 amino-terminal peptide in the PrP 27-30 control digest (33\% of the base peak intensity) to that found in the cross-linked PrP 27-30 dimer digest (11\% of the base peak intensity) suggests that as much as $33 \%$ of the cross-linked dimers have at least one free amino terminus, and must be cross-linked at sites undetected in this study. The observation of trimers and higher-order oligomers in the reaction mixtures substantiates this conclu- sion: obviously, these species must include at least one crosslink other than the one characterized. Significant effort was expended searching for additional cross-linked peptides. For example, in addition to searching for all amine-amine crosslinks, we also calculated the masses of all amine-Tyr and Tyr-Tyr cross-links. The existence of none of the thousands of possible cross-links could be substantiated. Also, the C-terminal GPI anchor is known to have two free amino groups, a primary amine in an ethanolamine, and a secondary amine in glucosamine; evidence of cross-links to either of these amines was not be observed. The lower yield of these products, and the necessity to work on small amounts of infectious materials isolated from the brains of diseased 


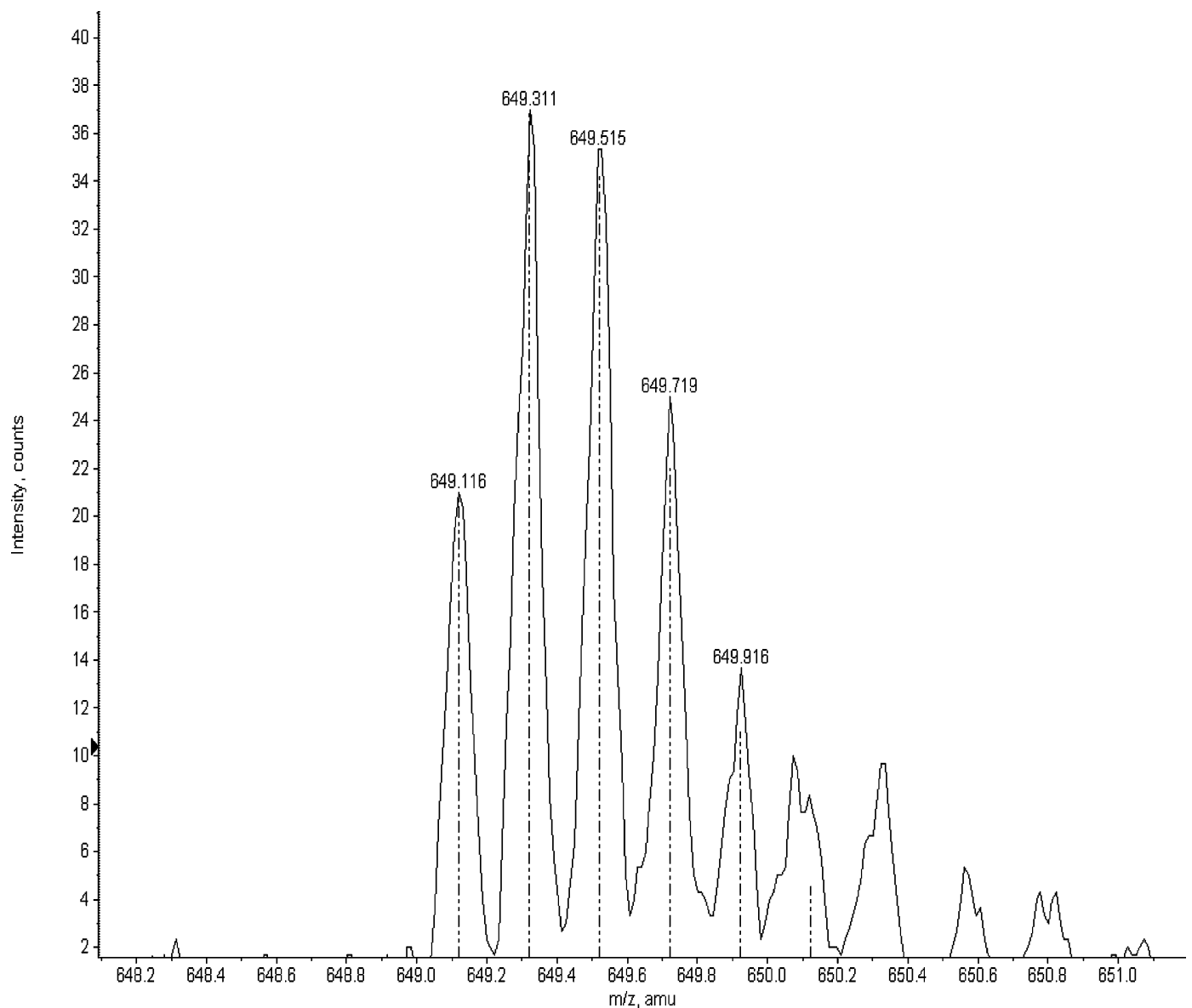

FIGURE 5: Experimental raw data (profile trace) for the Gly90-Lys101-X-Gly90-Lys106 cross-link as observed by nanoLC-ESI-QqTOF mass spectrometry and the theoretical isotope distribution (bar graph) calculated for the predicted elemental composition of $\mathrm{C}_{139} \mathrm{H}_{214} \mathrm{~N}_{47} \mathrm{O}_{44}$.

animals, are without doubt the experimental causes of this problem. The top-down FT-MS approach may provide a technical solution.

\section{DISCUSSION}

Acquiring some knowledge, even if limited, of the structure of $\mathrm{PrP}^{\mathrm{Sc}}$ is essential in understanding the infectious nature of this protein. Given the difficulties in applying conventional analytical approaches, such as NMR or X-ray crystallography, as a consequence of the insoluble and polymeric nature of $\mathrm{PrP}^{\mathrm{Sc}}$, the design of novel, unconventional analytical approaches appears to be an attractive option. Our study shows that the combination of chemical crosslinking, proteolytic digestion, and mass spectrometry, an approach that has been applied to the structural study of other monomeric and polymeric proteins $(15-24)$, can be successfully applied to $\operatorname{PrP}^{\mathrm{Sc}}$. Using $\mathrm{BS}^{3}$, an agent that reacts primarily with amino groups, PrP 27-30 was readily crosslinked with formation of dimers, trimers, and higher-order oligomers. This behavior contrasts with that of recombinant Syrian hamster PrP(90-231), known to be monomeric, whose reaction with $\mathrm{BS}^{3}$ did not result in any production of dimers, even at high concentrations of $\mathrm{BS}^{3}$ (data not shown). On the other hand, Riesner and co-workers have shown that an oligomeric, $\beta$-sheet rich preparation of recombinant PrP(90-231) made by careful control of the concentration of SDS, is readily cross-linked by $\mathrm{BS}^{3}(29)$. The identification of the amino acids that are involved in these cross-links has

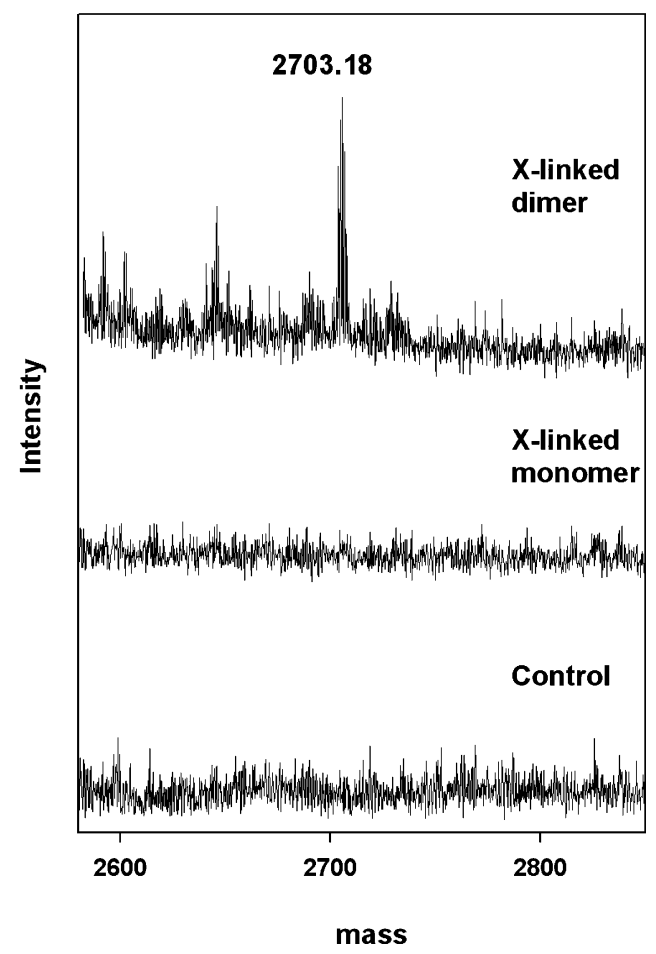

FIGURE 6: Detection of a cross-linked peptide, Gly90-Lys101$\mathrm{X}$-Gly90-Lys101, in the lys C digest of $\mathrm{BS}^{3}$-cross-linked PrP 2730 by MALDI-TOF. Bands were excised from gels and digested in gel with lys $\mathrm{C}$ overnight at $37^{\circ} \mathrm{C}$. Peptides were extracted, dried in vacuo, and analyzed by MALDI-TOF as described. 
not yet been established, and the recombinant material that was used was not infectious.

For a meaningful interpretation of our data, it is worth considering in detail the nature of our working material. The conjunction of detergent-containing buffers and PK treatment results in isolation of $\operatorname{PrP} 27-30$ in the form of large fibrillar aggregates known as "rods" (14). Such material contains virtually no other protein besides PrP, as assessed by SDSPAGE followed by Coomassie staining or, alternatively, mass spectrometry after proteolytic digestion (Figures 1 and 2). Such preparations also contain lipids and other nonproteinaceous material, but as far as these components have not interfered in our analyses, we refer to our preparations as "PrP 27-30". It is also important to note that PrP 27-30 rods do not constitute a homogeneous material and that during its manipulation (e.g., sonication, etc.) they can break and partially disaggregate, generating a fraction that has been rendered PK-sensitive (30). This material must not be confused with the $\mathrm{PK}$-sensitive $\mathrm{PrP}^{\mathrm{Sc}}$ subfraction present in tissues, mentioned in the introductory section and obviously excluded during preparation of PrP 27-30. The centrifugation step we used to recover PrP 27-30 after the crosslinking reaction ensures that lighter disaggregated material, potentially suffering partial denaturation, was excluded from our analyses.

Cross-linked PrP 27-30 oligomers represent exceptionally valuable sources of structural information from which insights can be obtained about how the monomers composing the $\mathrm{PrPSc}^{\mathrm{Sc}}$ aggregate are arranged, and which domains are near one another. In-gel proteolytic digestion followed by mass spectrometric analysis of PrP 27-30 cross-linked dimers consistently revealed the presence of an intermolecular crosslink involving two Gly90 amino termini. This means that in the PrP 27-30 aggregate, some if not all PrP monomers are arranged such that their Gly90 amino termini are close together. The maximum distance of two such $\mathrm{BS}^{3}$-linked amino groups, which is dictated by the length of the spacer arm of the cross-linking reagent, is $11.4 \AA$ (15).

Recent reports have described studies of two-dimensional crystals present in preparations of PrP 27-30 using electron crystallography $(31,32)$. The images that were obtained were interpreted as trimers of PrP 90-231 featuring parallel $\beta$-helices. The proposed model stacks all the $\beta$-helix-based trimers in a head-to-tail fashion to form the PrP 27-30 fibers. This is a quite reasonable assumption, since the alternative stacking of two trimers to form a hexamer with head-tohead contact would require a fibril to have two completely different interfaces (head-to-head and tail-to-tail). Furthermore, the proposed fibril with an exclusive head-to-tail orientation of trimers can readily accommodate continued growth by additional binding of PrP subunits.

The two-dimensional crystals have diameters of $\sim 69 \AA$ based on the dimensions of the unit cell (31). For a trimer with $P 3$ symmetry, the distance between equivalent atoms on the surface of adjacent monomers would be $72 \AA$, or $33 \%$ of the circumference of the trimer. It seems impossible for a cross-linker like $\mathrm{BS}^{3}$, which when fully extended spans only $11.4 \AA$, to accommodate such a distance to cross-link adjacent monomers in a trimer. However, the distance in the model between equivalent atoms in monomers oriented vertically along the axis of the fiber is only $14 \AA$. This is because the $\beta$-helical model of the monomer has three strands per monomer, and (3 strands) $\times(4.7 \AA /$ strand $)=14 \AA$. Cross-linking of two vertically stacked monomers would be possible as long as each amino termini could accommodate a motion of $\sim 2-3 \AA$. Such motion seems to be quite probable since proteinase $\mathrm{K}$ is able to hydrolyze amides six single bonds away from Gly90 to make amino-terminal Gly92, suggesting amino-terminal flexibility of Gly90, Gln91, and Gly92. Still, the $\mathrm{BS}^{3}$ distance constraint is more consistent with a helix containing only two strands per monomer (see Figure 7). The observed Gly90-Gly90 crosslink with $\mathrm{BS}^{3}$ further suggests that the monomer in one row of a fibril must be oriented directly (or nearly directly) above the monomer in an adjacent row. This is exactly what is expected of stacking $\beta$-helices atop each other to maximize backbone hydrogen bond formation.

In the proposed PrP 27-30 model (32), the Gly90 amino termini lie in the same plane, near the $P 3$ axis of symmetry, with an $\mathrm{N}-\mathrm{N}$ distance of $13 \AA$, which is indeed compatible with the $11.4 \AA$ distance constraint found in this study. It should be noted, however, that the amino termini in the model are located near the center of the assembly at the trimer-trimer interface, a location with what appears to be limited accessibility to solvent or reagent. Granted, if three deep clefts existed in the trimer-trimer interface, $\mathrm{BS}^{3}$ could have access to Gly90; however, such a cleft would diminish the contact area between two trimers and thereby destabilize the interaction. It appears to us that facile cross-linking by $\mathrm{BS}^{3}$ could be most easily accomplished by unraveling onethird of a helical turn (starting at the amino terminus) and reorienting the strand toward the exterior of the fibril, placing Gly90 on the surface of the fibril. Furthermore, in the published literature of the reaction of $\mathrm{BS}^{3}$ with model proteins of known structure, we are aware of no cases in which reactive lysine amino groups are not located on the surface of the protein. Presumably, the size and polarity of the sulfated $N$-hydroxysuccinimide leaving groups of $\mathrm{BS}^{3}$ prevent reaction with residues that are not on the exterior of the protein. Unraveling a full turn of the helix would result in only two strands per monomer, and the predicted distance between Gly90 amino termini in vertically adjacent monomers would be $9.4 \AA$, closer to the $11.4 \AA \mathrm{N}-\mathrm{N}$ distance of fully extended $\mathrm{BS}^{3}$.

The observation of tryptic peptide $221-229$ with Tyr226 esterified to suberic acid is good evidence that Tyr226 and the remaining C-terminal residues, Arg230 and Ser231, are all exposed to solvent. This is in agreement with models of PrP 27-30 in which N-terminal residues (90-171) adopt $\beta$-sheet secondary structure and form the core of the fibril, whereas the $\mathrm{B}$ and $\mathrm{C}$ helices (residues 172-231) maintain the same globular domain structure as found in $\operatorname{PrP}^{C}$ and are located on the exterior of the fibril. NMR data show that both Tyr225 and Tyr226 are on the surface of $\operatorname{PrP}^{\mathrm{C}}$. However, it is unclear why Tyr226 is so much more reactive to $\mathrm{BS}^{3}$ than Tyr225.

While we were successful in obtaining a significant yield of $\operatorname{PrP} 27-30$ dimers using $\mathrm{BS}^{3}$, in our hands this reagent also generated a substantially high yield of PrP 27-30 molecules with amino groups derivatized by suberic acid. Similar outcomes have been described by other authors. D'Ambrosio et al. (19) treated recombinant porcine aminoacylase 1 with $\mathrm{BS}^{3}$ and obtained cross-linked monomers and dimers; after SDS-PAGE separation and in-gel digestion 


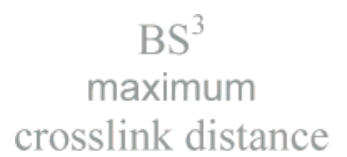

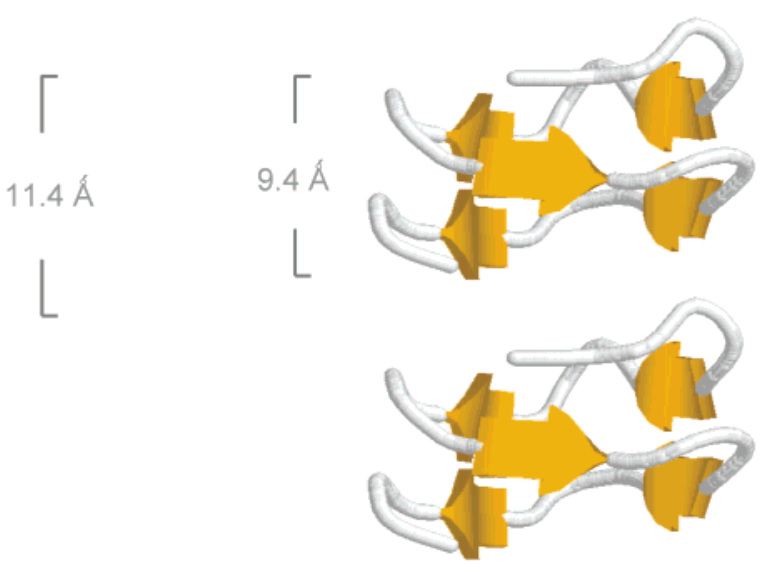

crossink distance 2-stranded helix
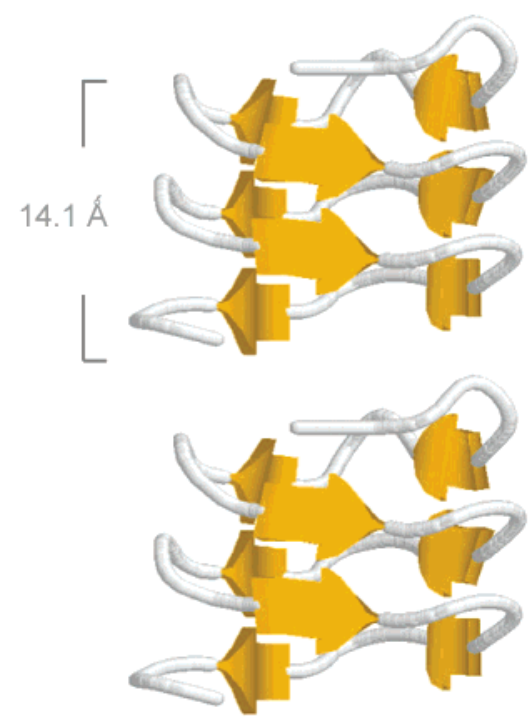

FIGURE 7: Gly90-Gly90 cross-link that is compatible with cross-linking of monomers stacked vertically along the fiber axis in a model of the core of PrP 27-30 based on stacking of $\beta$-helices. Coordinates for the model are from the $\beta$-helical protein $N$-acetylglucosamine acyltransferase (PDB entry 11xa). This figure was made with Protein Explorer (version 2.45).

with trypsin, MALDI analysis of the cross-linked monomer allowed detection of five peptides as their suberic acid amides (linkage via the $\epsilon$-amino group of lysines in the peptides). Several of these modified peptides were also detected in the cross-linked dimer band, which was also found to contain an intermolecular cross-link formed by bridging two peptides of identical sequence with $\mathrm{BS}^{3}$. As in our study, no intramolecular cross-links were found. Pearson et al. report similar difficulties in cross-linking cytochrome $c$ and RNase A, and discuss the widely different reactivity of different proteins with respect to cross-linking reagents (16). In our case, this difficulty is made worse since PrP 27-30, dispersed as a homogeneous suspension of prion rods in $1 \%$ sarkosyl (14), is known to be a compact structure. Location of the large Asn-linked glycans on the outside of the fiber would exacerbate this problem and explain the preference of $\mathrm{BS}^{3}$ in reacting with the amino-terminal portion, and minimize the level of formation of intramolecular cross-links.

In summary, we have successfully applied an analytical approach based on cross-linking and mass spectrometry to PrP 27-30, and obtained new structural information. Our data provide the first reported distance constraint for $\mathrm{PrP}^{\mathrm{Sc}}$ indicating the relative position of specific amino acid residues within PrP 27-30. Future application of our experimental approach should yield valuable information that, in combination with other novel approaches such as electron crystallography, molecular dynamics, and modeling, could eventually be integrated in a more complete model of the structure of $\mathrm{PrP}^{\mathrm{Sc}}$.

\section{ACKNOWLEDGMENT}

We thank Prof. José Cabezas-Cerrato (School of Medicine, University of Santiago) for continued support and encouragement. We greatly appreciate a critical review of the manuscript by Dr. Holger Wille. Thanks also to Dr. John Ericson for scripts written to assist in interpretation of the MS data and to Harold K. Cox for assistance in the preparation of figures.

\section{SUPPORTING INFORMATION AVAILABLE}

A table listing theoretical and experimental molecular weights of tryptic peptides from PrP 27-30, figures showing an expanded view of Figure 2, selected ion chromatograms for the major tryptic peptides of $\operatorname{PrP} 27-30$ obtained by nanoLC-ESI-QqTOF mass spectrometry, the partial sequence of ShaPrP, and confirmation of the proposed cross-link involving two Gly90 amino termini in cross-linked PrP 2730 by MS/MS. This material is available free of charge via the Internet at http://pubs.acs.org.

\section{REFERENCES}

1. Roepstorff, P., and Fohlman, J. (1984) Proposal for a common nomenclature for sequence ions in mass spectra of peptides, Biomed. Mass Spectrom. 11, 601.

2. Prusiner, S. B. (1982) Novel proteinaceous infectious particles cause scrapie, Science 216, 136-144.

3. Prusiner, S. B. (1998) Prions, Proc. Natl. Acad. Sci. U.S.A. 95, $13363-13383$.

4. Soto, C., and Castilla, J. (2004) The controversial protein-only hypothesis of prion propagation, Nat. Med. Suppl. 7, S63-S67.

5. Aguzzi, A., and Polymenidou, M. (2004) Mammalian prion biology: One century of evolving concepts, Cell 116, 313-327.

6. Prusiner, S. B. (1991) Molecular biology of prion diseases, Science $252,1515-1522$.

7. Come, J. H., Fraser, P. E., and Lansbury, P. T., Jr. (1993) A kinetic model for amyloid formation in the prion diseases: Importance of seeding, Proc. Natl. Acad. Sci. U.S.A. 90, 5959-5963.

8. Riek, R., Hornemann, S., Wider, G., Billeter, M., Glockshuber, R., and Wuthrich, K. (1996) NMR structure of the mouse prion protein domain $\operatorname{PrP}(121-321)$, Nature 382, 180-182.

9. Caughey, B. W., Dong, A., Bhat, K. S., Ernst, D., Hayes, S. F., and Caughey, W. S. (1991) Secondary structure analysis of the 
scrapie-associated protein $\operatorname{PrP} 27-30$ in water by infrared spectroscopy, Biochemistry 30, 7672-7680.

10. Stahl, N., Baldwin, M. A., Teplow, D. B., Hood, L., Gibson, B. W., Burlingame, A. L., and Prusiner, S. B. (1993) Structural studies of the scrapie prion protein using mass spectrometry and amino acid sequencing, Biochemistry 32, 1991-2002.

11. Requena, J. R., Groth, D., Legname, G., Stadtman, E. R., Prusiner, S. B., and Levine, R. L. (2001) Copper-catalyzed oxidation of the recombinant $\mathrm{SHa}(29-231)$ prion protein, Proc. Natl. Acad. Sci. U.S.A. 98, 7170-7175.

12. Safar, J., Wille, H., Itri, V., Groth, D., Serban, H., Torchia, M., Cohen, F. E., and Prusiner, S. B. (1998) Eight prion strains have $\mathrm{PrP}^{\mathrm{Sc}}$ molecules with different conformations, Nat. Med. 4, 11571165.

13. Tzaban, S., Friedlander, G., Schonberger, O., Horonchick, L., Yedidia, Y., Shaked, G., Gabizon, R., and Taraboulos, A. (2002) Protease-sensitive scrapie prion protein in aggregates of heterogeneous sizes, Biochemistry 41, 12868-12875.

14. McKinley, M. P., Meyer, R. K., Kenaga, L., Rahbar, F., Cotter, R., Serban, A., and Prusiner, S. B. (1991) Scrapie prion rod formation in vitro requires both detergent extraction and limited proteolysis, J. Virol. 65, 1340-1351.

15. Young, M. M., Tang, N., Hempel, J. C., Oshiro, C. M., Taylor, E. W., Kuntz, I. D., Gibson, B. W., and Dollinger, G. (2000) High throughput protein fold identification by using experimental constraints derived from intramolecular cross-links and mass spectrometry, Proc. Natl. Acad. Sci. U.S.A. 97, 5802-5806.

16. Pearson, K. M., Pannell, L. K., and Fales, H. M. (2002) Intramolecular cross-linking experiments on cytochrome $c$ and ribonuclease A using an isotope multiplet method, Rapid Commun. Mass Spectrom. 16, 149-159.

17. Sinz, A. (2003) Chemical cross-linking and mass spectrometry for mapping three-dimensional structures of proteins and protein complexes, J. Mass Spectrom. 38, 1225-1237.

18. Rossi, V., Gaboriaud, C., Lacroix, M., Ulrich, J., Fontecilla-Camps, J. C., Gagnon, J., and Arlaud, G. J. (1995) Structure of the catalytic region of human complement protease C1s: Study by chemical cross-linking and three-dimensional homology modeling, Biochemistry 34, 7311-7321.

19. D’Ambrosio, C., Talamo, F., Vitale, R. M., Amodeo, P., Tell, G., Ferrara, L., and Scaloni, A. (2003) Probing the dimeric structure of porcine aminoacylase 1 by mass spectrometric and modeling procedures, Biochemistry 42, 4430-4443.

20. Schulz, D. M., Ihling, C., Clore, G. M., and Sinz, A. (2004) Mapping the topology and determination of a low-resolution threedimensional structure of the calmodulin-melittin complex by chemical cross-linking and high-resolution FTICRMS: Direct demonstration of multiple binding modes, Biochemistry 43, 47034715 .
21. Cai, K., Itoh, Y., and Khorana, H. G. (2001) Mapping of contact sites in complex formation between transducin and light-activated rhodopsin by covalent crosslinking: Use of a photoactivatable reagent, Proc. Natl. Acad. Sci. U.S.A. 98, 4877-4882.

22. Kawasaki, H., Nose, T., Muta, T., Iwanaga, S., Shimohigashi, Y., and Kawabata, S. (2000) Head-to-tail polymerization of coagulin, a clottable protein of the horseshoe crab, J. Biol. Chem. 275, 35297-35301.

23. Back, J. W., Artal Sanz, M., De Jong, L., De Koning, L., Nijtmans, L. G. J., De Koster, C. G., Grivell, L. A., Van der Spek, H., and Muijsers, A. O. (2002) A structure of the yeast prohibitin complex: Structure prediction and evidence from chemical crosslinking and mass spectrometry, Protein Sci. 11, 2471-2478.

24. Egnaczyk, G. F., Greis, K. D., Stimson, E. R., and Maggio, J. E. (2001) Photoaffinity cross-linking of Alzheimer's disease amyloid fibrils reveals interstrand contact regions between assembled $\beta$-amyloid peptide subunits, Biochemistry 40, 11706-11714.

25. Diringer, H., Beekes, M., Özel, M., Simon, D., Queck, I., Cardone, F., Pocchiari, M., and Ironside, J. W. (1997) Highly infectious purified preparations of disease-specific amyloid of transmissible spongiform encephalopathies are not devoid of nucleic acids of viral size, Intervirology 40, 238-246.

26. Laemmli, U. K. (1970) Cleavage of structural proteins during the assembly of the head of bacteriophage T4, Nature 227, 680685 .

27. Shevchenko, A., Wilm, M., Vorm, O., and Mann, M. (1996) Mass spectrometric sequencing of proteins silver-stained polyacrylamide gels, Anal. Chem. 68, 850-858.

28. Leavell, M. D., Novak, P., Behrens, C. R., Schoeniger, J. S., and Kruppa, G. H. (2004) Strategy for selective chemical cross-linking of tyrosine and lysine residues, J. Am. Soc. Mass Spectrom. 15, 1604-1611.

29. Jansen, K., Schäfer, O., Birkmann, E., Post, K., Serban, H., Prusiner, S. B., and Riesner, D. (2001) Structural intermediates in the putative pathway from the cellular prion protein to the pathogenic form, Biol. Chem. 382, 683-691.

30. Wille, H., Prusiner, S. B., and Cohen, F. E. (2000) Scrapie infectivity is independent of amyloid staining properties of the N-terminally truncated prion protein, J. Struct. Biol. 130, 323338.

31. Wille, H., Michelitsch, M. D., Guénebaut, V., Supattapone, S., Serban, A., Cohen, F. E., Agard, D. A., and Prusiner, S. B. (2002) Structural studies of the scrapie prion protein by electron crystallography, Proc. Natl. Acad. Sci. U.S.A. 99, 3563-3568.

32. Govaerts, C., Wille, H., Prusiner, S. B., and Cohen, F. E. (2004) Evidence for assembly of prions with left-handed $\beta$-helices into trimers, Proc. Natl. Acad. Sci. U.S.A. 101, 8342-8347. 